\title{
Full-field hard X-ray microscopy based on aberration-corrected Be CRLs
}

\author{
Andreas Schropp $^{\mathrm{a}}$, Dennis Brückner ${ }^{\mathrm{a}, \mathrm{b}}$, Jessica Bulda ${ }^{\mathrm{a}}$, Gerald Falkenberg ${ }^{\mathrm{a}}$, Jan Garrevoet ${ }^{\mathrm{a}}$, \\ Johannes Hagemann ${ }^{\mathrm{a}}$, Frank Seiboth ${ }^{\mathrm{a}}$, Kathryn Spiers ${ }^{\mathrm{a}}$, Frieder Koch ${ }^{\mathrm{c}}$, Christian David ${ }^{\mathrm{c}}$, \\ Marianna Gambino ${ }^{\mathrm{d}}$, Martin Veselýd ${ }^{\mathrm{d}}$, Florian Meirer ${ }^{\mathrm{d}}$, and Christian G. Schroer ${ }^{\mathrm{a}, \mathrm{e}}$

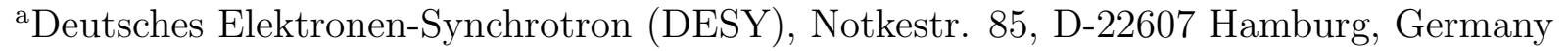 \\ ${ }^{\mathrm{b}}$ Ruhr Universität Bochum, Universitätsstr. 150, D-44780 Bochum, Germany \\ ${ }^{c}$ Paul Scherrer Institut (PSI), CH-5232 Villigen, Switzerland \\ ${ }^{d}$ Utrecht University, Universiteitsweg 99, NL-3584 CG, Utrecht, The Netherlands \\ eUniversität Hamburg, Luruper Chaussee 149, D-22761 Hamburg, Germany
}

\begin{abstract}
To date, compound refractive X-ray lenses made out of Beryllium (Be CRLs) have been seldom applied for full-field microscopy with high spatial resolution, which was probably due to residual aberrations of these optics. However, in combination with the recent development of made-to-measure phase plates, the typical spherical aberration of beryllium compound refractive lenses (Be CRLs) can now be completely removed. In this way, distortion-free images of a sample are obtained, which is especially important for tomographic applications. First full-field imaging experiments with aberration-corrected Be CRLs were carried out at beamline P06 at the synchrotron radiation X-ray source PETRA III (DESY Hamburg, Germany). In order to maximize the magnification of the X-ray microscope for full-field microscopy, the full length of the beamline combining the micro- and nanohutch was utilized, enabling a large sample-to-detector distance. In this contribution, we present first imaging results, demonstrating the potential of Be CRLs for direct high-resolution X-ray tomography.
\end{abstract}

Keywords: hard X-ray microscopy, full-field imaging, tomography, refractive X-ray optics, compound refractive lenses, corrective phase plates

\section{INTRODUCTION}

Full-field X-ray microscopy is a powerful technique to investigate the structure of specimens with high spatial resolution. ${ }^{1-3}$ Due to the short wavelength of X rays and their relatively weak interaction with matter especially in the hard X-ray regime, internal structural information can be revealed by tomographic applications in 3D. ${ }^{4-6}$ Compound refractive lenses made out of Beryllium (Be CRLs) ${ }^{7-9}$ are well established optics in the hard X-ray regime and are commonly employed at various synchrotron radiation X-ray sources as well as X-ray free-electron lasers (XFELs). ${ }^{10,11}$ They can be used for full-field X-ray microsocopy as well as focusing optics in scanning X-ray experiments. ${ }^{12}$ However, in order to achieve an optimal performance X-ray optics generally have to be manufactured with a high precision on the nanometer length scale, which is in many cases very challenging to realize mechanically. ${ }^{13}$ CRLs are typically produced by an embossing process and small errors of the CRL-shape lead to aberrations in X-ray imaging experiments. Probably for this reason CRLs have been rarely used for full-field X-ray microscopy with high spatial resolution up to now.

The recent development of correction phase plates can overcome these issues since they are easier to fabricate and can be inserted directly behind a lens stack. In a similar way as normal glasses in the optical regime these phase plates are created for specific X-ray lenses made-to-measure in order to perfectly remove residual aberrations yielding a diffraction-limited performance of the optics. ${ }^{14,15}$ In the following, we present first fullfield imaging results using aberration-corrected Be CRLs measured at beamline P06 at the synchrotron radiation X-ray source PETRA III (DESY Hamburg, Germany).

Send correspondence to A. Schropp. E-mail: schropp@xray-lens.de, telephone: +49 (0)40 89986537

\footnotetext{
X-Ray Nanoimaging: Instruments and Methods IV, edited by Barry Lai,

Andrea Somogyi, Proc. of SPIE Vol. 11112, 1111208 · (C) 2019 SPIE

CCC code: $0277-786 \mathrm{X} / 19 / \$ 21 \cdot$ doi: $10.1117 / 12.2528422$
} 


\section{ABERRATION CORRECTION}

In a preceding experiment at beamline P06 the optics were characterized by scanning coherent X-ray microscopy (ptychography) at an X-ray energy of $12 \mathrm{keV}$. In this measurement a nano-structured sample with features sizes between $50 \mathrm{~nm}$ to $200 \mathrm{~nm}$ was positioned close to the focal plane of the lens stack. The sample was structured in a $1 \mu \mathrm{m}$-thick tungsten layer deposited on a CVD diamond substrate. ${ }^{11}$ The lens stack consisted of 50 single Be CRLs, each with a radius of curvature of $R=50 \mu \mathrm{m}$ and geometric aperture of $D=300 \mu \mathrm{m}$. At the X-ray photon energy of $E=12 \mathrm{keV}$ the focal length of the whole stack was $f=221 \mathrm{~mm}$. The sample was then scanned within an area of $2 \mu \mathrm{m} \times 2 \mu \mathrm{m}$ in $20 \times 20$ steps with a step size of $100 \mathrm{~nm}$. At each scan point a diffraction pattern was measured by an Eiger X 4M-detector (Dectris Ltd.) at a distance of $L=6.85 \mathrm{~m}$ from the sample. Using a standard ptychographic reconstruction algorithm $(\mathrm{ePIE})^{16}$ the complex-valued illumination and transmission function of the object was reconstructed from the set of 441 diffraction patterns $(21 \times 21$ scan points $)$ as shown in Fig. 1a) and $1 \mathrm{~b}$ ), respectively. ${ }^{15}$ The method yields the complete information of the nano-focused X-ray beam, ${ }^{17}$ including the full beam caustic as indicated in Fig. $1 \mathrm{c}$ ).
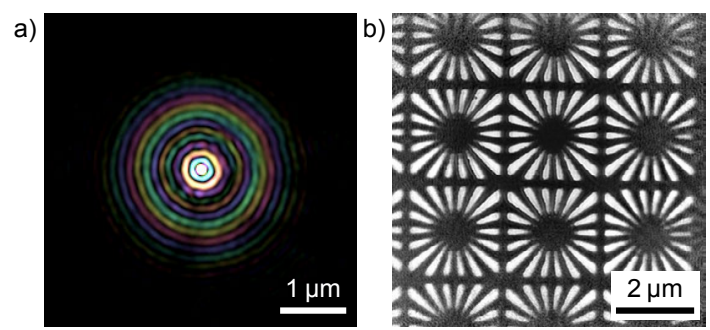

c)

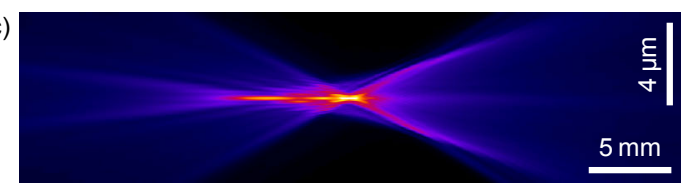

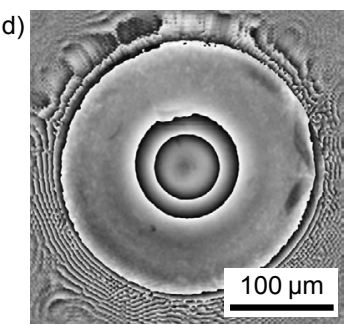

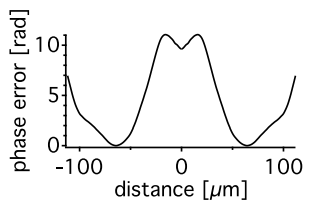

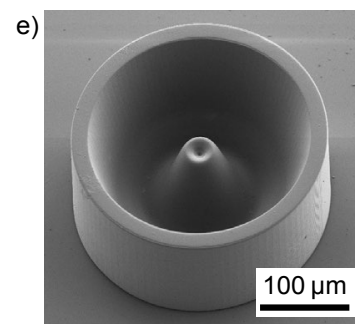

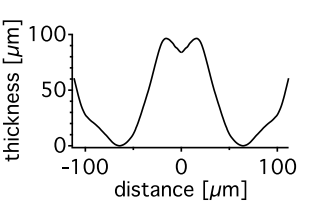

Figure 1. Characterization of a stack of 50 Be CRLs by ptychography. a) Retrieved illumination function. b) Reconstructed transmission function of the nano-structured sample (phase distribution). c) Caustic of the nano-focused beam showing the typical spherical aberraction of Be CRLs. d) Phase errors of the exit wave directly after the optics obtained from the reconstructed illumination function. e) SEM image of the phase plate fabricated by additive manufacturing.

From the reconstructed illumination function the exit wave field after the Be CRLs can be obtained by numerically propagating the reconstructed wave field by a distance of $191 \mathrm{~mm}$ towards the lens. An additional subtraction of the corresponding spherical wave reveals phase errors of the Be CRLs as illustrated in Fig. 1d). After phase unwrapping and azimuthal averaging the radial phase error can be obtained [cf. lower plot in Fig. 1d)], which is the basis to build the corrective phase plate made-to-measure. In this case, additive manufacturing was used to fabricate the phase plate in a polymer by 3D-printing (Nanoscribe $\mathrm{GmbH}$ ).

In order to correct the spherical aberration of the optics, the thickness of the phase plate $t$ has to vary as $t=\frac{1}{k \delta} \cdot \varphi_{\mathrm{err}}=\frac{\lambda}{2 \pi \delta} \cdot \varphi_{\mathrm{err}}$, where $k=\frac{2 \pi}{\lambda} \approx 61000 \mathrm{\mu m}^{-1}$ is the wave number and $\delta=1.885 \cdot 10^{-6}$ the refractive index decrement of the polymer at the X-ray energy of $E=12 \mathrm{keV}(\lambda=1.03 \AA)$. Inserting the polymer phase plate as an additional optical element in the vicinity of the optics then introduces a phase shift of $\varphi=-k \delta t$, perfectly compensating the lens errors. An SEM-image of the final phase plate is shown in Fig. 1e) and the corresponding radial depth profile in the plot below.

\section{FULL-FIELD MICROSCOPY}

Be CRLs were implemented at beamline P06 as an objective lens for full-field X-ray microscopy. The KirkpatrickBaez mirror system (KB mirrors) available in the microhutch of P06 was used as condenser optics to enhance the photon flux and the angular divergence of the incoming X-ray beam. The sample was then imaged with the previously characterized lens consisting of 50 individual Be CRLs with $R=50 \mu \mathrm{m}$ as outlined in Sec. 2. The manufactured phase plate was positioned closely in front of the main lens stack. In Fig. 2 a sketch of the imaging 
geometry is shown. Furthermore, a rotating diffuser plate out of boron carbide was introduced in front of the $\mathrm{KB}$ mirrors in order to reduce the spatial coherence and to create a homogeneous illumination. Therefore, the projection images measured on the X-ray detector represent the absorption contrast within the sample.

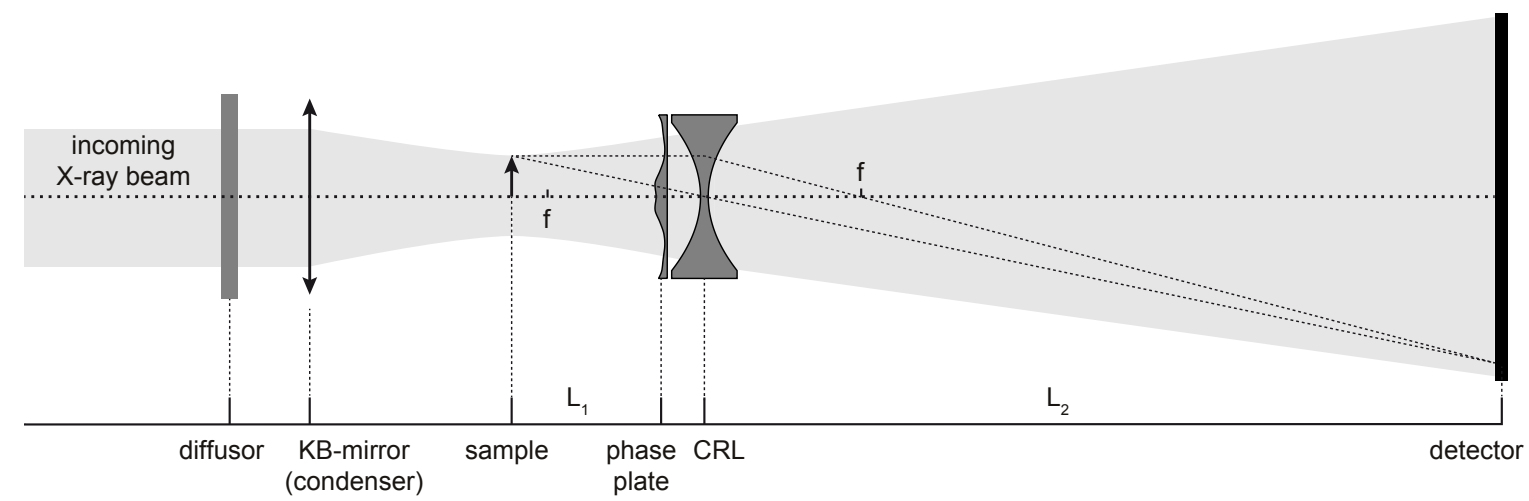

Figure 2. Full-field imaging geometry as implemented at beamline P06 (PETRA III).

For a fixed sample-to-lens distance $L_{1}$ a sharp image of the sample is formed at a lens-to-detector distance of $L_{2}=L_{1} f /\left(L_{1}-f\right)$, where $f$ is the focal length of the lens. ${ }^{4}$ The spatial resolution is limited by diffraction to $d_{t}=0.75 \lambda / 2 N A$ with the numerical aperture $N A$ given by $N A=D_{\text {eff }} / 2 L_{1}$. The parameter $D_{\text {eff }}$ describes the effective aperture of the lens, which is typically slightly smaller than the geometric aperture of the lens due to absorption within the lens. The depth of focus $d_{l}$ is given by $d_{l}=0.64 \lambda /(N A)^{2}$ and the total magnification $m$ of the imaging setup by $m=L_{2} / L_{1}$. The phase plate is inserted closely in front of the lens thereby removing the spherical aberration of the Be CRLs and yielding distortion-free images of a sample.

\section{EXPERIMENTAL SETUP}

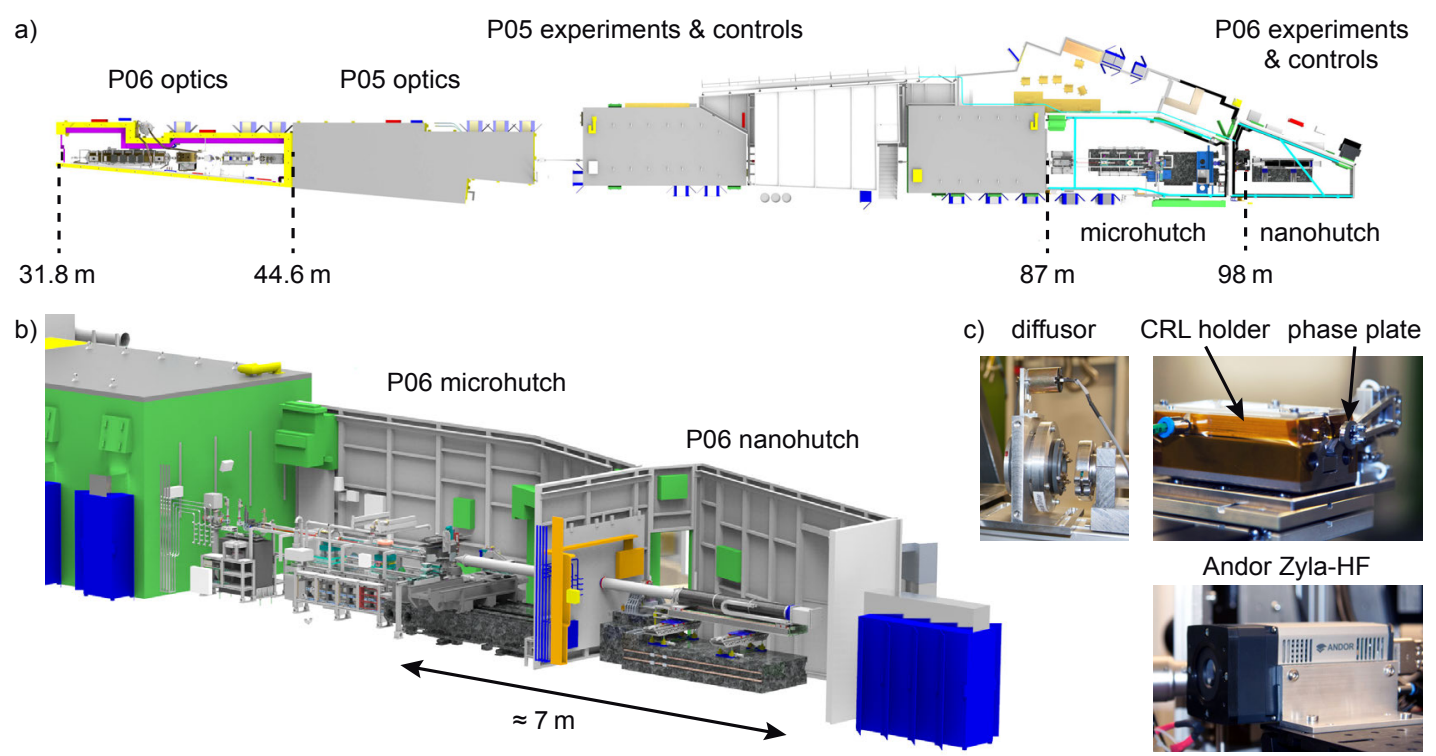

Figure 3. a) General layout of beamline P06 at PETRA III. b) Beamline P06 has two separate experimental hutches for the micro- and nanoprobe end stations. Running in combined operation mode using both hutches at the same time the distance between sample and detector can be maximized to about $7 \mathrm{~m}$. c) Images of the boron carbide diffusor, the CRL lens holder with movable phase plate and the Andor Zyla-HF detector. 
Beamline P06 at PETRA III is optimized for hard X-ray scanning microscopy with high spatial resolution. ${ }^{18}$ It has two experimental hutches, the micro- and nanohutch, typically offering distinct focusing setups such as a Kirkpatrick-Baez mirror system for moderate focusing to about $250 \mathrm{~nm}$ in the microhutch and mainly nanofocusing refractive lenses (NFLs), ${ }^{19,20}$ Fresnel zone plates (FZPs) ${ }^{21,22}$ and multi-layer lenses (MLLs) ${ }^{23-25}$ for tighter focusing towards $50 \mathrm{~nm}$ and below in the nanohutch. The beamline shares sector 4 of PETRA III (low- $\beta$ section) with the imaging beamline P05 in the Max-von-Laue hall (cf. Fig. 3).

At beamline P06 the distance between the X-ray source and a sample positioned in the microprobe is about $95 \mathrm{~m}$. The X-ray beam is monochromatized using a channelcut monochromator, higher harmonics of the undulator radiation are suppressed using the horizontal offset mirrors (HO-mirrors), and the photon flux can be enhanced for full-field microscopy by using additional pre-focusing lenses available in the optics hutch of P06. In this case, the experiment was carried out using the micro- and nanohutch in combined operation mode, i. e., sample and objective lens were positioned in the micro- and the detector in the nanohutch. In this way, a larger distance between sample and detector of $L_{2}=6850 \mathrm{~mm}$ could be realized to maximize the magnification of the microscope. The sample-to-lens distance was $L_{1}=228.5 \mathrm{~mm}$ for this specific lens stack with a focal length of $f=221.1 \mathrm{~mm}$ at an X-ray energy of $12 \mathrm{keV}$. The spatial resolution of the optics is limited by diffraction to about $d_{t}=70 \mathrm{~nm}$, with $D_{\text {eff }}=242.6 \mu \mathrm{m}$ and $N A=5.5 \times 10^{-4}$ (cf. Sec. 3). In this case, however, the spatial resolution was mainly limited by the pixel size $p$ of the Andor Zyla-HF detector of $p=6.5 \mu \mathrm{m}$, providing an effective pixel size of $p_{\text {eff }}=p / m \approx 220 \mathrm{~nm}$, with $m=L_{2} / L_{1} \approx 30$ the magnification in the given imaging geometry.

\section{RESULTS}

The performance of the microscope was investigated at the example of imaging a resolution test chart (Siemensstar) and a number of catalytic particles. The Siemens-star was manufactured by NTT-AT (ATN/XRESO-50HC) with $500 \mathrm{~nm}$ thick tantalum structures having $50 \mathrm{~nm}$ thinnest lines and spaces. A direct comparison of imaging performance without and with the phase plate is shown in Figs. 4 a) and 4 b), respectively. The images show the average of 30 exposures with $0.3 \mathrm{~s}$ exposure time each and are flat- and background corrected. They were recorded in exactly the same geometric configuration but only the phase plate was inserted in the latter case. Especially looking at the smaller structures or the numbers the improvement in imaging quality is visible in terms of image sharpness and contrast.
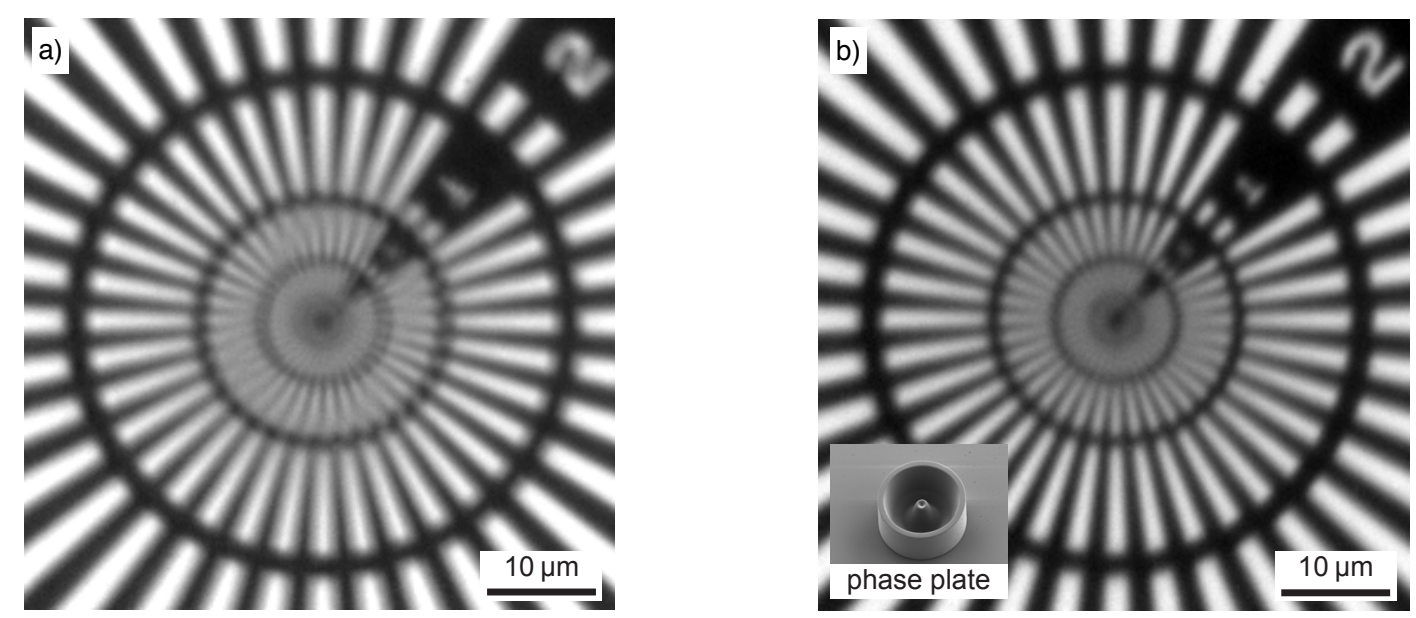

Figure 4. a) Full-field image obtained with a stack of 50 Be CRLs. b) Same as in a) but using additionally the 3D-printed phase plate (cf. inset).

In Fig. 5 a summary of a tomographic experiment carried out on fluid-catalytic-cracking (FCC) particles is shown. They were contained in a quartz capillary suitable for in-situ experiments. The tomographic scan was recorded with 1200 steps with a step size $0.15^{\circ}$ and an exposure time of $0.3 \mathrm{~s}$ per image. However, about $10^{\circ}$ of the full range of $180^{\circ}$ could not be measured due to the capillary mount blocking the X-ray beam in this angular 
range [cf. gap in sinogram in Fig. 5 b)]. The missing angular range leads to some artifacts in the tomographic reconstruction by filtered back projection visible in Fig. $5 \mathrm{c}$ ).
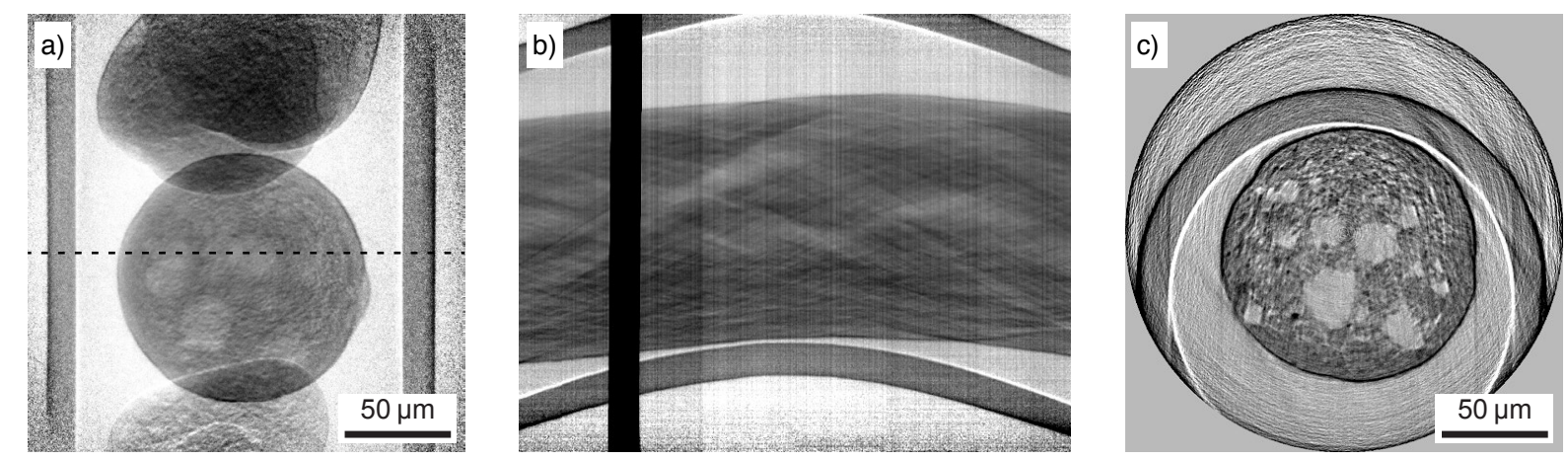

Figure 5. a) Full-field projection image of different FCC-particles embedded within a quartz capillary. b) Sinogram used for the tomographic reconstruction shown in c). The dashed horizontal line in Fig. a) indicates the vertical position of the tomographic slice.

Nevertheless, although the experiments indicate the high potential of using diffraction-limited Be CRLs for full-field microscopy in the hard X-ray regime, the maximum possible spatial resolution could not be achieved yet. In the currently possible geometry and at the given magnification, the pixel size of the detector is the main limiting factor. In future experiments a photon-efficient detector with smaller pixels will resolve this issue and potentially allow to increase the spatial resolution of full-field X-ray tomography with Be CRLs towards $50 \mathrm{~nm}$ with optimal performance.

\section{CONCLUSIONS}

We demonstrated that Be CRLs in combination with a phase plate made-to-measure are suitable X-ray optics for full-field X-ray microscopy in the hard X-ray regime at high spatial resolution. The additional phase plate corrects the pronounced spherical aberrations of the optics and improves the quality of the measured projection images. It reduces image artifacts and enhances the contrast. At beamline P06 the full-field microscopy option is interesting for fast tomographic pre-characterization if several samples are to be investigated and good candidates are then chosen for a subsequent scanning X-ray fluorescence (XRF) experiment. Switching to scanning microscopy using the KB-mirror system can be easily accommodated by moving the pre-focusing optics, diffusor and objective lens out of the X-ray beam. In this way, even combined XRF and ptychography imaging measurements can be carried out in a subsequent step.

In order to reach the highest possible resolution of about $50 \mathrm{~nm}$ for these specific optics and optimal tomography results, the imaging geometry and the detector need to be adapted to yield a higher magnification and a smaller effective pixel size. In addition, software developments are required for the tomographic reconstuction taking into account absorption effects within the Be CRLs. ${ }^{26}$

\section{ACKNOWLEDGEMENTS}

We acknowledge DESY (Hamburg, Germany), a member of the Helmholtz Association HGF, for the provision of experimental facilities. Parts of this research were carried out at beamline P06 of PETRA III.

\section{REFERENCES}

[1] Schroer, C. G., Meyer, J., Kuhlmann, M., Benner, B., Günzler, T. F., Lengeler, B., Rau, C., Weitkamp, T., Snigirev, A., and Snigireva, I., "Nanotomography based on hard x-ray microscopy with refractive lenses," Appl. Phys. Lett. 81(8), 1527-1529 (2002).

[2] Bosak, A., Snigireva, I., Napolskii, K. S., and Snigirev, A., "High-Resolution Transmission X-ray Microscopy: A New Tool for Mesoscopic Materials," Advanced Materials 22(30), 3256-3259 (2010). 
[3] Byelov, D. V., Meijer, J.-M., Snigireva, I., Snigirev, A., Rossi, L., van den Pol, E., Kuijk, A., Philipse, A., Imhof, A., van Blaaderen, A., Vroege, G. J., and Petukhov, A. V., "In situ hard X-ray microscopy of self-assembly in colloidal suspensions," RSC Advances 3(36), 15670 (2013).

[4] Lengeler, B., Schroer, C., Tümmler, J., Benner, B., Richwin, M., Snigirev, A., Snigireva, I., and Drakopoulos, M., "Imaging by parabolic refractive lenses in the hard x-ray range," J. Synchrotron Rad. 6, 1153-1167 (1999).

[5] Lengeler, B., Schroer, C. G., Richwin, M., Tümmler, J., Drakopoulos, M., Snigirev, A., and Snigireva, I., "A microscope for hard x-rays based on parabolic compound refractive lenses," Appl. Phys. Lett. 74(26), 3924-3926 (1999).

[6] Weiß, D., Schneider, G., Niemann, B., Guttmann, P., Rudolph, D., and Schmahl, G., "Computed tomography of cryogenic biological specimens based on X-ray microscopic images," Ultramicroscopy 84, 185-197 (2000).

[7] Snigirev, A., Kohn, V., Snigireva, I., and Lengeler, B., "A compound refractive lens for focusing high energy x-rays," Nature (London) 384, 49 (1996).

[8] Schroer, C. G., Lengeler, B., Benner, B., Tümmler, J., Günzler, T. F., Drakopoulos, M., Simionovici, A. S., Snigirev, A., and Snigireva, I., "Compound refractive lenses for x-ray microanalysis," AIP Conference Proceedings 507, 694-699 (2000).

[9] Lengeler, B., Schroer, C. G., Benner, B., Günzler, T. F., Kuhlmann, M., Tümmler, J., Simionovici, A. S., Drakopoulos, M., Snigirev, A., and Snigireva, I., "Parabolic refractive X-ray lenses: A breakthrough in X-ray optics," Nucl. Instrum. Methods A 467-468, 944-950 (2001).

[10] Lengeler, B., Schroer, C. G., Kuhlmann, M., Benner, B., Günzler, T. F., Kurapova, O., Zontone, F., Snigirev, A., and Snigireva, I., "Refractive x-ray lenses," J. Phys. D: Appl. Phys. 38, A218-A222 (2005).

[11] Schropp, A., Hoppe, R., Meier, V., Patommel, J., Seiboth, F., Lee, H. J., Nagler, B., Galtier, E. C., Arnold, B., Zastrau, U., Hastings, J. B., Nilsson, D., Uhlen, F., Vogt, U., Hertz, H. M., and Schroer, C. G., "Full spatial characterization of a nanofocused x-ray free-electron laser beam by ptychographic imaging," Scientific Reports 3, 1633 (2013).

[12] Schroer, C. G., Kuhlmann, M., Günzler, T. F., Benner, B., Kurapova, O., Patommel, J., Lengeler, B., Roth, S. V., Gehrke, R., Snigirev, A., Snigireva, I., Stribeck, N., Almendarez-Camarillo, A., and Beckmann, F., "Full-field and scanning microtomography based on parabolic refractive x-ray lenses," Proc. SPIE 6318, $63181 \mathrm{H}(2006)$.

[13] Chen, Y.-T., Lo, T.-N., Chu, Y. S., Yi, J., Liu, C.-J., Wang, J.-Y., Wang, C.-L., Chiu, C.-W., Hua, T.-E., Hwu, Y., Shen, Q., Yin, G.-C., Liang, K. S., Lin, H.-M., Je, J. H., and Margaritondo, G., "Full-field hard x-ray microscopy below $30 \mathrm{~nm}$ : a challenging nanofabrication achievement," Nanotechnology 19(39), 395302 (2008).

[14] Seiboth, F., Schropp, A., Scholz, M., Wittwer, F., Rödel, C., Wünsche, M., Ullsperger, T., Nolte, S., Rahomäki, J., Parfeniukas, K., Giakoumidis, S., Vogt, U., Wagner, U., Rau, C., Boesenberg, U., Garrevoet, J., Falkenberg, G., Galtier, E. C., Lee, H. J., Nagler, B., and Schroer, C. G., "Perfect x-ray focusing via fitting corrective glasses to aberrated optics," Nat. Comm. 8, 14623 (2017).

[15] Schropp, A., Bruckner, D., Bulda, J., Falkenberg, G., Garrevoet, J., Seiboth, F., Wittwer, F., Koch, F., David, C., and Schroer, C. G., "Scanning Hard X-Ray Microscopy Based on Be CRLs," Microscopy and Microanalysis 24(S2), 186-187 (2018).

[16] Maiden, A. M. and Rodenburg, J. M., "An improved ptychographical phase retrieval algorithm for diffractive imaging," Ultramicroscopy 109(10), 1256-1262 (2009).

[17] Schropp, A., Boye, P., Feldkamp, J. M., Hoppe, R., Patommel, J., Samberg, D., Stephan, S., Giewekemeyer, K., Wilke, R. N., Salditt, T., Gulden, J., Mancuso, A. P., Vartanyants, I. A., Weckert, E., Schöder, S., Burghammer, M., and Schroer, C. G., "Hard x-ray nanobeam characterization by coherent diffraction microscopy," Appl. Phys. Lett. 96(9), 091102 (2010).

[18] Schroer, C. G., Boye, P., Feldkamp, J. M., Patommel, J., Samberg, D., Schropp, A., Schwab, A., Stephan, S., Falkenberg, G., Wellenreuther, G., and Reimers, N., "Hard X-ray nanoprobe at beamline P06 at PETRA III," Nucl. Instrum. Meth. A 616(2), 93-97 (2010). 
[19] Schroer, C. G., Kuhlmann, M., Hunger, U. T., Günzler, T. F., Kurapova, O., Feste, S., Frehse, F., Lengeler, B., Drakopoulos, M., Somogyi, A., Simionovici, A. S., Snigirev, A., Snigireva, I., Schug, C., and Schröder, W. H., "Nanofocusing parabolic refractive x-ray lenses," Appl. Phys. Lett. 82(9), 1485-1487 (2003).

[20] Schroer, C. G., Kurapova, O., Patommel, J., Boye, P., Feldkamp, J., Lengeler, B., Burghammer, M., Riekel, C., Vincze, L., van der Hart, A., and Küchler, M., "Hard x-ray nanoprobe based on refractive x-ray lenses," Appl. Phys. Lett. 87(12), 124103 (2005).

[21] Vila-Comamala, J., Jefimovs, K., Raabe, J., Pilvi, T., Fink, R. H., Senoner, M., Maassdorf, A., Ritala, M., and David, C., "Advanced thin film technology for ultrahigh resolution X-ray microscopy," Ultramicroscopy 109(11), 1360-1364 (2009).

[22] Mohacsi, I., Vartiainen, I., Guizar-Sicairos, M., Karvinen, P., Guzenko, V. A., Müller, E., Kewish, C. M., Somogyi, A., and David, C., "Fabrication and characterization of high-efficiency double-sided blazed x-ray optics," Optics Letters 41(2), 281 (2016).

[23] Morgan, A. J., Prasciolu, M., Andrejczuk, A., Krzywinski, J., Meents, A., Pennicard, D., Graafsma, H., Barty, A., Bean, R. J., Barthelmess, M., Oberthuer, D., Yefanov, O., Aquila, A., Chapman, H. N., and Bajt, S., "High numerical aperture multilayer Laue lenses," Scientific Reports 5(1), 9892 (2015).

[24] Kubec, A., Melzer, K., Gluch, J., Niese, S., Braun, S., Patommel, J., Burghammer, M., Leson, A., and IUCr, "Point focusing with flat and wedged crossed multilayer Laue lenses," J. Synchrotron Rad. 24(2), 413-421 (2017).

[25] Bajt, S., Prasciolu, M., Fleckenstein, H., Domaracký, M., Chapman, H. N., Morgan, A. J., Yefanov, O., Messerschmidt, M., Du, Y., Murray, K. T., Mariani, V., Kuhn, M., Aplin, S., Pande, K., Villanueva-Perez, P., Stachnik, K., Chen, J. P., Andrejczuk, A., Meents, A., Burkhardt, A., Pennicard, D., Huang, X., Yan, H., Nazaretski, E., Chu, Y. S., and Hamm, C. E., "X-ray focusing with efficient high-NA multilayer Laue lenses," Light: Science \& Applications 7(3), 17162 (2018).

[26] Schroer, C. G., Benner, B., Günzler, T. F., Kuhlmann, M., Lengeler, B., Rau, C., Weitkamp, T., Snigirev, A., and Snigireva, I., "Magnified hard X-ray microtomography: Toward tomography with sub-micron resolution," Proc. SPIE 4503, 23-33 (2002). 